\title{
Relationship between chewing ability and nutritional status in Japanese older adults: A cross- sectional study
}

\author{
Keiko Motokawa1*, Yurie Mikami1, Maki Shirobe1, Ayako Edahiro1, Yuki Ohara1, Masanori \\ Iwasaki1, Yutaka Watanabe2, Hisashi Kawai1, Takeshi Kera3, Shuichi Obuchi1, Yoshinori \\ Fujiwara1, Kazushige Ihara4, Hirohiko Hirano1 \\ 1 Tokyo Metropolitan Institute of Gerontology, Tokyo, Japan \\ 2 Hokkaido University, Hokkaido, Japan \\ 3 Takasaki University of Health and Welfare, Gunma, Japan \\ 4 Hirosaki University, Aomori, Japan \\ * Correspondence: Keiko Motokawa kemotokawa@gmail.com; Tokyo Metropolitan Institute of Gerontology, \\ 35-2 Sakae-cho, Itabashi-Ku, Tokyo 173-0015, Japan; tel +81 339643241 Ext 4215; fax +81 339642316
}

\begin{abstract}
Objectives: This study aimed to determine the relationship between objective chewing ability and nutritional status of Japanese community-dwelling elders. Design: Cross sectional study. Participants: In total, 509 community-dwelling elders living in the Tokyo metropolitan area participated in a comprehensive survey occurring in October 2013. Measurements: Basic characteristics referred to sex, age, and body mass index. Undernutrition was examined through serum albumin levels. Chewing ability was examined through a color-changeable xylitol gum. By evaluating color changes in the chewing gum. Nutritional intake was examined through the semiquantitative Food Frequency Questionnaire. Results: In the poor chewing ability group, all nutrient intake levels were significantly low, except for carbohydrates and all food groups intake levels were significantly low, except for cereals, confectionery, sugars, seasonings, and spices. Additionally, after adjusting for covariates, chewing ability showed a significant association with undernutrition. Conclusion: We concluded that chewing ability was closely associated with nutrient and different food groups intake as well as undernutrition among Japanese community-dwelling elders. Thus, in order to ensure a comprehensive nutritional management, nutritionists and dentists should collaborate when treating the same patients.
\end{abstract}

Keywords: nutrient intake, food groups, chewing ability

\section{Introduction}

Japan is experiencing unprecedented populational aging, transforming the country into a superaged society; accordingly, by 2015, the proportion of the population over 65 years of age had reached $26.0 \%$ [1]. Thus, aging developed countries - like Japan - urgently need to take measures toward providing appropriate care that meets the needs of these elders, who incur in increased illness incidences owing to their advanced age. In Health Japan 21 (the 2nd term), a basic document used to promote the comprehensive implementation of national health interventions, the percentage of community-dwelling elders with undernutrition (i.e. a body mass index [BMI] of less than is approximately $18 \%$; nonetheless, this number is expected to increase in the future together with continued populational aging [2]. Moreover, especially among the elderly, undernutrition is related to reduced cure rates, increased risk of complications, and death [3-5]. Thus, there is an urgent need for promoting early interventions aimed at improving Japanese elders' eating behaviors to ensure that they have suitable meals and avoid undernutrition.

Recently, the term oral frailty has been utilized to help identify people that require early stage interventions aimed at diminishing their frailty. To have oral frailty, people need to have a low rating 
on more than three of the following six oral status: Number of natural teeth, chewing ability, articulatory oral motor skill, tongue pressure, and subjective difficulties in eating and swallowing [6]. Having oral frailty has been reported to increase the risk of needing long-term care, of sarcopenia, of death, and one's physical frailty [6]. Moreover, decreased chewing ability has been associated with life function deterioration, being housebound, bedridden, and sarcopenia onset in communitydwelling elderly women [7]. Against this background, we aim to discuss the relationship between Japanese elders' chewing ability decline and food intake.

Additionally, impaired dentition among the elderly has been reported to be significantly associated with nutrition intake and intake of different food groups; for example, individuals who have impaired dentition have reduced protein, sodium, potassium, calcium, vitamin A, vitamin E, and dietary fiber intake levels compared to those without impaired dentition [8]. Zhu et al [9] analyzed data from the American National Health and Nutrition Examination Survey, which was conducted among nearly 9000 individuals (25\% of which had over 65 years of age); after adjusting for socioeconomic factors (i.e. age, gender, race, income, physical activity, and smoking status), the authors found that people with 20 teeth or less had a lower intake of fruits and vegetables compared to people with over 28 teeth, as well as a lower nutrient intake, missing minerals, proteins, dietary fibers, and most vitamins. Thus, ensuring that people maintain their tooth number seems important for food intake promotion. Notwithstanding, at present, it is not possible to directly intervene in this number to enhance its related outcomes. Furthermore, these studies $[8,9]$ did not investigate other possible influencing factors (e.g. undernutrition) and the condition of functional teeth (i.e. the presence or absence of dentures).

Therefore, this study aimed to cross-sectionally investigate the relationship between chewing ability - which was objectively evaluated - and nutritional status of Japanese community-dwelling elders. Specifically, we aimed to analyze the relationship between chewing ability and undernutrition. We aimed to provide the literature with further evidence that may help in the promotion and development of early interventions aimed at preventing undernutrition.

\section{Materials and Methods}

\subsection{Participants}

The survey was conducted in October 2013 among community-dwelling elderly in urban areas of Tokyo, Japan. Figure 1 delineates recruitment details. Among 1,471 people that were invited to participate, 791 agreed to participate and were included in the study. Of these, 509 participants (294 women, $57.8 \%$ ) were analyzed, since it was necessary to exclude three that missed the chewing ability evaluation and 279 who did not participate in the eating survey.

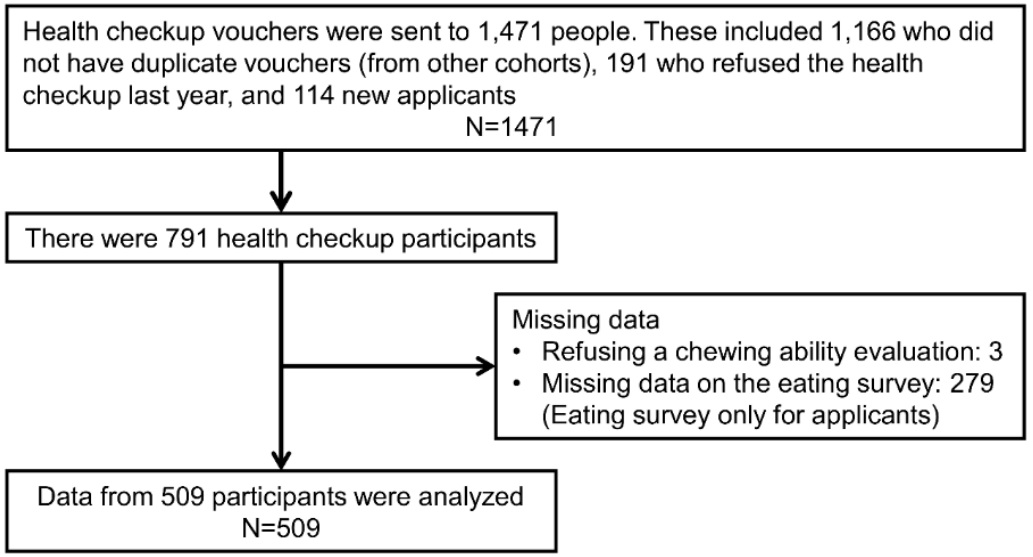

Figure 1 Recruitment of Research Participants

2.2. Survey Items

2.3. Basic Information 
A nurse or a trained researcher verbally asked each participant about their gender, age and instrumental activity of daily living (IADL, as assessed by the Tokyo Metropolitan Institute of Gerontology-Index of Competence: TMIG-IC) [10], cognitive function (Mini-Mental State Examination: MMSE) [11] and their medical history (high blood pressure, stroke, heart disease, diabetes, hyperlipidemia). Moreover, a dentist or dental hygienist conducted a check for each participant's number of functional teeth, including dentures.

\subsection{Evaluation of Chewing Ability}

In order to examine participants' chewing ability, we used a color-changeable chewing gum (i.e. a gum used to evaluate masticatory performance Xylitol; Lotte, Saitama, Japan). This procedure comprised asking each participant to chew the gum for one minute; afterwards, participants were supposed to spit the gum into a pouch. In order to evaluate chewing ability, we used a color chart that was divided into five levels [12], the chewing gum was green, but turned red when chewed; if the color of the gum was close to green, the chewing ability was evaluated as low. In this study, levels 1, 2, and 3 were classified as "poor," and levels 4 and 5 were classified as "good." Participants were thus classified into the poor chewing group and the good chewing group.

\subsection{Body Measurements}

We measured participants' height, weight, and calculated their BMI.

\subsection{Undernutrition Diagnosis}

In order to examine undernutrition, participants had their blood drawn and were subjected to evaluation-using blood serum albumin values. For this analysis, the value of $4.0 \mathrm{~g} / \mathrm{dL}$ of albumin was designated as the cutoff for classifying participants into two groups: The undernutrition and healthy groups [13].

\subsection{Eating Survey}

The eating survey was administered to consenting participants. In order to evaluate participants' nutrient and food group intake, we used the semi-quantitative Food Frequency Questionnaire (FFQ), which was developed by Takahashi et al [14]. This questionnaire is based on 29 food groups, and it was conducted by a nutritionist. Participants were asked about their food intake over the past month, food portion sizes, and the amount of food eaten in one meal. The residual correction method was used, with energy intake from nutrients and food group intake figures [15]. Light colored vegetables were mushrooms, and beverages included tea, coffee, juice, soft drinks, alcohol, and more.

\subsection{Statistical Analysis}

We conducted comparative analyses for chewing ability using an independent t-test for continuous variables and a Chi-squared test for categorical variables. Furthermore, in order to calculate the deficiency ratio of the poor chewing group, we used the ratio of the good chewing group as the standard; then, we compared participants' differences in nutrient and food group intake by chewing ability. In addition, we examined the relationship between chewing ability and undernutrition adjusting for BMI and number of functional teeth. Finally, in order to analyze the relation between undernutrition and chewing ability, we used a logistic regression analysis. Statistical analyses were conducted using SPSS 25.0, and significance was set at less than $5 \%$.

\subsection{Ethical Considerations}

This study was conducted with approval from the Ethics Committee of the Tokyo Metropolitan Geriatric Hospital and Institute of Gerontology (approval No. 2013, 1253). Prior to participation, all participants and their legal representatives (e.g. family members) were provided with an oral/written explanation about study objectives, methods, and expected outcomes, and provided informed consent. We affirm that we have taken all measures to ensure data anonymity and masking. 


\section{Results}

Participants' characteristics by chewing ability are displayed in Table 1. In total, 301 participants (59.1\%) had poor and 208 had good chewing ability (40.9\%). Moreover, 60 participants $(11.8 \%)$ presented undernutrition, whereas $449(88.2 \%)$ had a good nutritional status.

Table 1 Subject Characteristics Compared to Chewing Ability

\begin{tabular}{|c|c|c|c|c|c|c|c|c|}
\hline \multirow{3}{*}{$\begin{array}{l}\text { Sex } \\
\text { Age }\end{array}$} & \multirow{3}{*}{$\begin{array}{l}\text { female } \\
\text { year }\end{array}$} & \multirow{2}{*}{\multicolumn{3}{|c|}{$\begin{array}{c}\text { Poor chewing } \\
\text { ability } \\
60.3 \%\end{array}$}} & \multicolumn{3}{|c|}{$\begin{array}{c}\text { Good chewing } \\
\text { ability }\end{array}$} & \multirow{2}{*}{ 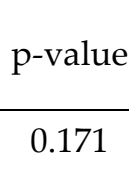 } \\
\hline & & & & & & & & \\
\hline & & 73.9 & \pm & 5.8 & 72.1 & \pm & 5.1 & $<0.001$ \\
\hline BMI & $\mathrm{kg} / \mathrm{m}^{2}$ & 23 & \pm & 3.1 & 23 & \pm & 3.3 & 0.964 \\
\hline Fat-free Mass Index & $\mathrm{kg} / \mathrm{m}^{2}$ & 16.1 & \pm & 1.6 & 16.4 & \pm & 2.1 & 0.113 \\
\hline Serum albumin & $\mathrm{g} / \mathrm{dl}$ & 4.28 & \pm & 0.2 & 4.34 & \pm & 0.2 & 0.001 \\
\hline TMIG-IC & score & 12.2 & \pm & 1.2 & 12.3 & \pm & 1.3 & 0.203 \\
\hline MMSE & score & 28.3 & \pm & 2.0 & 28.7 & \pm & 1.6 & 0.071 \\
\hline Functional teeth & teeth & 26.3 & \pm & 4.1 & 27.6 & \pm & 1.8 & $<0.001$ \\
\hline Hypertension & positive & & & & & & & 0.205 \\
\hline Stroke & positive & & & & & & & 0.517 \\
\hline Cardiovascular disease & positive & & & & & & & 0.538 \\
\hline Diabetes & positive & & & & & & & 0.355 \\
\hline Hyperlipidemia & positive & & & & & & & 0.703 \\
\hline $\begin{array}{l}\text { Chronic obstructive } \\
\text { pulmonary disease }\end{array}$ & positive & & & & & & & 1.000 \\
\hline Cancer & positive & & & & & & & 0.425 \\
\hline Depression & positive & & & & & & & 0.099 \\
\hline
\end{tabular}

For continuous variables, we used t-test; for categorical variables, we used the $\chi^{2}$-test.

Table 2 shows participants' nutrient and food group intake by chewing ability. In the poor chewing ability group, energy, protein, fat, calcium, iron, vitamin A, vitamin D, vitamin B1, vitamin B2 and vitamin C had intake levels that were significantly low, except for carbohydrates, thus including all food groups. In addition, the poor chewing ability group had intake levels that were significantly low in contrast, such as potatoes, vegetables, seaweed, beans, fish, meat, eggs, milk, fruits, usual drinks (such as coffee, tea soft drinks and alcohol), nuts, seeds and fats and oils. Figure 2 presents a comparison of food group intake with nutrient intake level according to masticatory function. If the good group is designated a score of 100, then in the poor group, nutrients with a value over $10 \%$ are protein, fats, iron, vitamin $\mathrm{A}$, and vitamin C. Food groups with a value over $10 \%$ are potatoes, yellow vegetables, seaweed, beans, seafood, meat, and nuts.

Table 2 Nutrient and Food Groups Intake Compared to Chewing Ability

\begin{tabular}{llrllrllc}
\hline & & \multicolumn{2}{c}{$\begin{array}{c}\text { Poor chewing } \\
\text { ability }\end{array}$} & \multicolumn{2}{c}{$\begin{array}{c}\text { Good chewing } \\
\text { ability }\end{array}$} & $\begin{array}{c}\text { p- } \\
\text { value }\end{array}$ \\
\hline Energy & kcal & 1859 & \pm & 486 & 2009 & \pm & 599 & 0.002 \\
Protein & $\mathrm{g}$ & 66.9 & \pm & 15.5 & 74.0 & \pm & 19.5 & $<0.001$ \\
Fat & $\mathrm{g}$ & 60.6 & \pm & 18.9 & 68.5 & \pm & 23.9 & $<0.001$ \\
Carbohydrate & $\mathrm{g}$ & 248.5 & \pm & 59.7 & 259.0 & \pm & 75.2 & 0.081
\end{tabular}




\begin{tabular}{|c|c|c|c|c|c|c|c|c|}
\hline Calcium & $\mathrm{g}$ & 641 & \pm & 172 & 705 & \pm & 216 & $<0.001$ \\
\hline Iron & $\mathrm{g}$ & 7.9 & \pm & 2.2 & 8.7 & \pm & 2.8 & $<0.001$ \\
\hline Vitamin A & $\mu g$ & 571 & \pm & 141 & 649 & \pm & 178 & $<0.001$ \\
\hline Vitamin D & $\mu g$ & 8.3 & \pm & 2.0 & 9.3 & \pm & 2.5 & $<0.001$ \\
\hline Vitamin B1 & $\mathrm{mg}$ & 0.9 & \pm & 0.3 & 1.0 & \pm & 0.3 & $<0.001$ \\
\hline Vitamin B2 & $\mathrm{mg}$ & 1.1 & \pm & 0.3 & 1.2 & \pm & 0.4 & $<0.001$ \\
\hline Vitamin C & $\mathrm{mg}$ & 104 & \pm & 28 & 119 & \pm & 35 & $<0.001$ \\
\hline Cereals & $\mathrm{kcal}$ & 601.5 & \pm & 68.8 & 600.4 & \pm & 86.6 & 0.871 \\
\hline Potatoes & kcal & 23.9 & \pm & 5.7 & 27.7 & \pm & 7.1 & $<0.001$ \\
\hline $\begin{array}{l}\text { Green-yellow } \\
\text { vegetables }\end{array}$ & kcal & 23.9 & \pm & 4.6 & 28.1 & \pm & 5.7 & $<0.002$ \\
\hline $\begin{array}{l}\text { Pale colored } \\
\text { vegetables }\end{array}$ & kcal & 36.1 & \pm & 8.2 & 42.2 & \pm & 10.4 & $<0.003$ \\
\hline Seaweed & kcal & 2.1 & \pm & 0.5 & 2.8 & \pm & 0.6 & $<0.004$ \\
\hline Pluses & kcal & 88.0 & \pm & 17.7 & 100.1 & \pm & 22.2 & $<0.005$ \\
\hline $\begin{array}{l}\text { Fish and } \\
\text { shellfish }\end{array}$ & kcal & 115.0 & \pm & 24.8 & 129.3 & \pm & 31.3 & $<0.006$ \\
\hline Meat & kcal & 159.1 & \pm & 49.4 & 195.6 & \pm & 62.2 & $<0.007$ \\
\hline Eggs & kcal & 39.3 & \pm & 7.2 & 42.1 & \pm & 9.0 & $<0.008$ \\
\hline $\begin{array}{l}\text { Milk } \\
\text { products }\end{array}$ & kcal & 156.4 & \pm & 34.4 & 168.4 & \pm & 43.3 & $<0.009$ \\
\hline Fruits & $\mathrm{kcal}$ & 70.6 & \pm & 20.7 & 80.1 & \pm & 26.1 & $<0.010$ \\
\hline Confectionery & $\mathrm{kcal}$ & 266.9 & \pm & 154.5 & 284.4 & \pm & 194.6 & 0.260 \\
\hline Usual drinks & kcal & 81.4 & \pm & 28.1 & 89.5 & \pm & 35.3 & 0.004 \\
\hline $\begin{array}{l}\text { Sugar and } \\
\text { sweeteners }\end{array}$ & kcal & 40.0 & \pm & 11.8 & 41.2 & \pm & 14.9 & 0.330 \\
\hline $\begin{array}{l}\text { Nuts and } \\
\text { seeds }\end{array}$ & kcal & 14.6 & \pm & 5.2 & 20.6 & \pm & 6.6 & $<0.001$ \\
\hline Fats and oils & $\mathrm{kcal}$ & 90.5 & \pm & 21.0 & 102.4 & \pm & 26.5 & $<0.001$ \\
\hline Seasoning & kcal & 55.7 & \pm & 13.4 & 54.9 & \pm & 16.9 & 0.560 \\
\hline
\end{tabular}

For continuous variables, we used t-test.
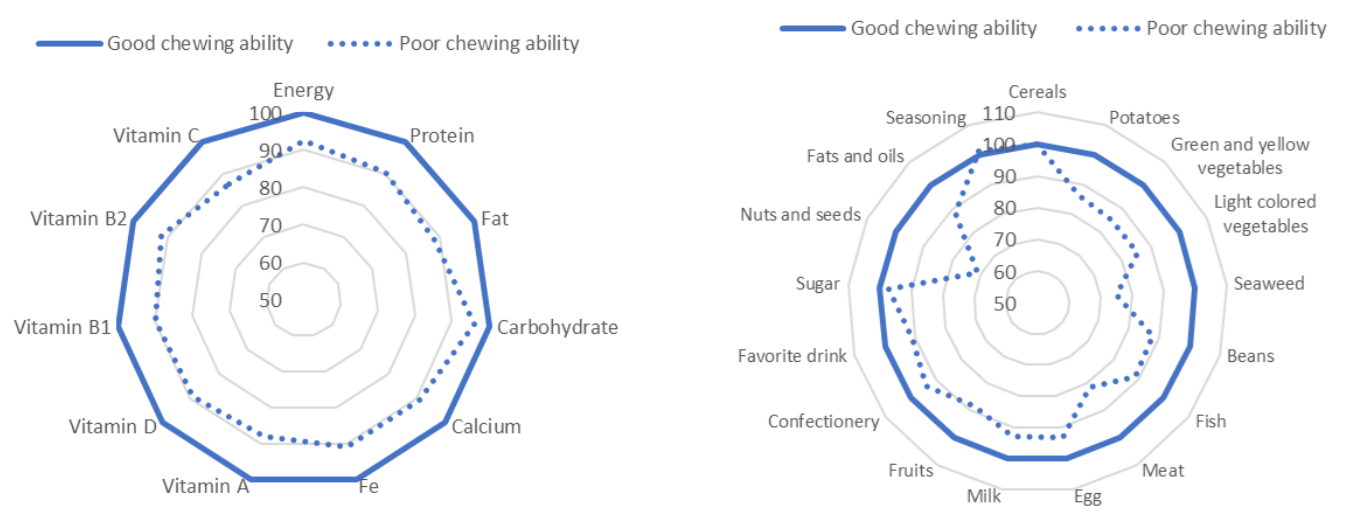

Figure 2. Difference from poor ability when good chewing ability is set to 100 . 
Figure 3 presents the association between chewing ability and undernutrition. In the poor chewing ability group, the undernutrition percentages were higher. Moreover, a logistic regression analysis regarding the relationship between chewing ability and undernutrition (Table 3) showed that, even when controlling for sex, age, TMIG-IC score, MMSE score, BMI, stroke, number of functional teeth, energy intake and protein intake, chewing ability was found to be independently and significantly correlated with undernutrition (OR:1.453, 95\% CI:1.004-2.022, $\mathrm{p}=0.027$ ).

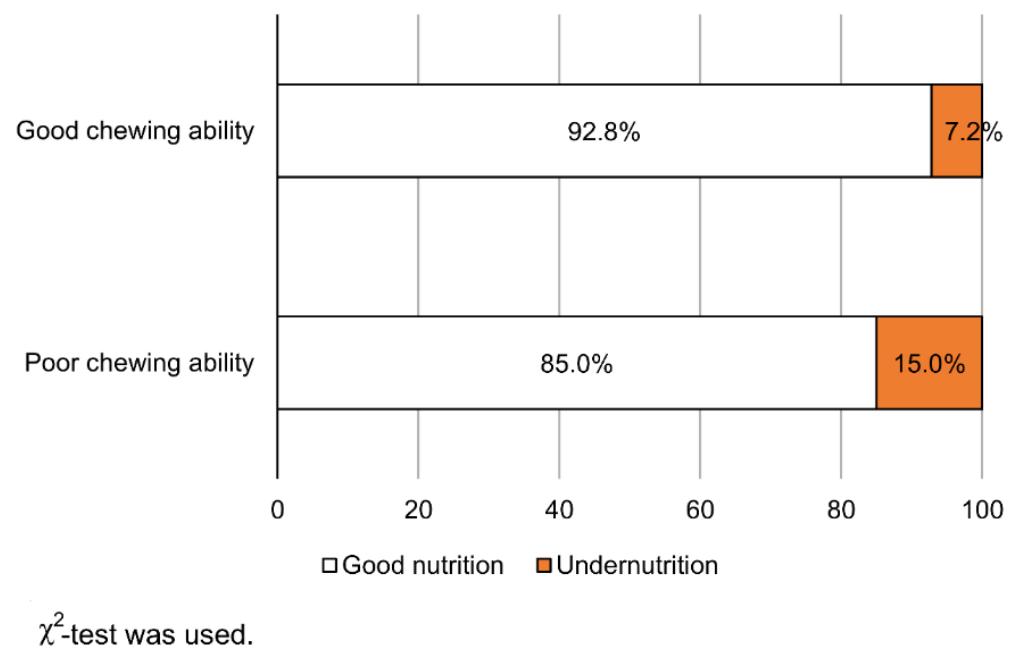

Figure 3. Rates of Undernutrition due to Differences in Chewing Ability

Table 3 Relationship between Chewing Ability and Nutritional Status

\begin{tabular}{cccc}
\hline & Odds ratio & $95 \%$ CI & p-value \\
\hline $\begin{array}{c}\text { Model 1 } \\
\text { Chewing ability (0: good, 1: poor) }\end{array}$ & 1.550 & $(1.205-1.994)$ & $<0.001$ \\
\hline $\begin{array}{c}\text { Model 2 } \\
\text { Chewing ability (0: good, 1: poor) }\end{array}$ & 1.453 & $(1.004-2.022)$ & 0.027 \\
\hline
\end{tabular}

Dependent variables: Serum albumin values (cut off: $4.0 \mathrm{mg} / \mathrm{dL}$; 0: healthy, $1:$ undernutrition). Model 1 was adjusted for sex and age. Model 2 adjusted for Model 1 plus the Tokyo Metropolitan Institute of Gerontology-Index of Competence score, Mini-Mental State Examination score, Body Mass Index, stroke, number of functional teeth, energy intake, and protein intake.

\section{Discussion}

Our results showed that chewing ability was related to nutrient and food group intake among community-dwelling elders in urban areas of Japan. Moreover, after adjusting for BMI and number of functional teeth, chewing ability was significantly related to undernutrition. Thus, good chewing ability among the elderly may help them maintain a high dietary variety; in turn, this can lead to nutritional status maintenance.

In the current study, chewing ability was associated with nutrient and food group intake and undernutrition even when adjusting for number of functional teeth-including dentures. Previous studies have shown that people with impaired dentition have substantially lower protein, sodium, potassium, vitamin A, vitamin E, and dietary fiber intake compared to those without it [8]. Additionally, as mentioned in the Introduction section, Zhu et al [9] analyzed data from 9000 American people and found that those with 20 teeth or less had lower fruit and vegetable intake 
compared to those with 28 teeth or more, even after adjusting for socioeconomic factors (e.g. age, gender, race, and income), physical activity, and smoking, had lower intakes of minerals, proteins, dietary fibers, and most vitamins. Nonetheless, although these studies have illustrated the importance of tooth number maintenance for food intake, interventions regarding tooth number, in itself, is currently not possible. Moreover, these studies have failed to investigate oral function and the presence or absence of dentures.

On the other hand, Inomata et al [16] compared peoples' tooth number with occlusal force exerted when chewing, and found that occlusal force was related to walking speed and the intake of green-yellow vegetables, seafood, dietary fiber, and vitamins. Furthermore, among people that have no teeth (i.e. full dentures), about $60 \%$ answered that they can still eat hard foods such as pickled radish or dried squid [17]. These results indicate that even full dentures contribute to the practical recovery of one's chewing ability. Additionally, according to results from the 2011 Survey of Dental Diseases, teeth retention among the Japanese elderly had greatly improved: The percentage of seniors over 80 years old who retain more than 20 of their own teeth reached $50 \%$ [18]. On the other hand, a study showed that the current measures taken to improve oral function, including swallowing, remain inadequate [19]. Therefore, our results seemed to support previous studies and show that understanding oral function is important for maintaining and improving nutritional status.

Our results also showed that, among the poor chewing ability group, all nutrient intake levels were significantly low, except for carbohydrates; and that intake levels for all food groups were significantly low, except for cereals, confectionery, sugars, seasonings, and spices. Among the elderly, the intake of protein derived from meat and seafood is especially important as a countermeasure to frailty. During old age, although muscle mass and functionality are naturally reduced, studies have shown that a causal factor of such diminishment relates to the anabolic suppression response of the skeletal muscle formation [20]; when compared with that during youth, it was shown to be weakened, and the reason may be that aging brings about protein anabolic resistance. Confirming, a study showed that, when a woman of frail status and aged over 65 years was fed a high-protein diet $(1.23 \mathrm{~g} / \mathrm{kg} /$ day $)$, her protein assimilation increased and her nitrogen equilibrium became positive, suggesting the effectiveness of a high protein diet for the elderly [21].

However, and recently, the importance of dietary variety, rather than the intake of single foods or nutrients, has also been highlighted. In our previous research, we awarded participants with one point for eating each of these ten foods every day [22]: Seafood, meat, eggs, milk, soybeans/soybean products, green-yellow vegetables, seaweed, potatoes, fruits, and fats/oils. Participants could score a maximum of 10 points, and if they did not eat any of these points, no points were awarded. In this study, we used the Kihon-checklist [23] criteria to distinguish between the frail, pre-frail, and robust and found that the frail group scored 3.9, the pre-frail 4.3, and the robust 4.5 points, corroborating a previous study reporting a significant relationship between participants' frailty levels and their Dietary Variety Score, even after adjusting for gender, age, drinking and smoking status, past medical history, blood serum albumin values, and energy intake. Moreover, dietary variety has been shown to associate with not just frailty but also cognitive function: A longitudinal study reported that high dietary variety reduces the risk of cognitive decline by $44 \%$ [24]. Varying food types may lead to an adequate intake of proteins, vitamins, and minerals which may contribute to the maintenance of muscle mass, muscle strength and cognitive function. Therefore, variation in feeding is an important intervention indicator nutritional support in elders. Nonetheless, our results showed that poor chewing ability may lead to reduced intake of protein (e.g. from meat and fish) and decreased dietary variety, both of which can potentially affect people's nutritional status, thus possibly leading to undernutrition. To put our results into perspective, our sample comprised Japanese elderly that were: relatively healthy; living independently in the community; and that chose, independently, to participate in the outpatient-style health check. Even in this population, there was an association between poor chewing ability and food intake and low nutrition, suggesting that earlier support and intervention may be necessary. Thus, in order to support Japanese elders' food intake, practitioners and stakeholders related to nutritional health of the elderly should endeavor to understand their oral 
function and denture status, and aim at providing cooperative care that unites nutrition and dental departments at earlier stages, rather than just offering nutritional guidance/meal support.

Recently, intervention studies have combined nutritional guidance, oral function improvement interventions, and the use of prosthetics; Bradbury et al [25] divided the study sample between a "complete dentures only" group and a "complete dentures with nutritional guidance" group, and reported that the second group experienced significant improvements intakes of vegetable and fruit. Moreover, Kikutani et al [26] divided a sample of people that required long-term care between the "meal support only" group and the "meal support with oral function training" group, showing that the serum albumin values of the second group rose significantly. Further corroborating, Suzuki et al [27] reported that the provision of simple nutritional support amid denture provision is effective in improving patients' nutritional intake and chewing ability. Thus, previous studies concur with our statement toward the need for nutrition and dentistry to collaborate, as this collaboration can contribute to the maintenance of Japanese elders' health: It may allow for more positive influences toward elders' healthy life expectancy, and similar influences may not be achievable by separately conducting oral management and nutritional support. Finally, we believe that future intervention studies aimed at examining the effects of the collaboration between nutrition and dental departments and at identifying the causal relationships between our studied variables are warranted.

Although we provided fruitful results for the literature, this research also had several limitations. First, we used a cross-sectional design, so our study cannot indicate causality. Second, as this survey required participants to go to the venue, our participants were relatively healthy; specially, the eating survey was conducted by consenting participants, implying that our sample may comprise a population with higher health and dietary literacy. Thus, future intervention and longitudinal studies aimed at identifying the causality in the relationship between objective chewing ability and nutrition are warranted.

\section{Conclusions}

Our results indicated a significant association between Japanese elders' chewing ability with nutrient and food group intake and undernutrition. Thus, nutrition management should be conducted with an understanding over and guided by patients' oral function and denture status; moreover, we highlight that nutritionists and dentists should endeavor to cooperate when treating the same patients, as their collaboration can produce better outcomes than their separate interventions. We think future longitudinal and intervention studies are warranted, and researchers should endeavor to identify possible effective interventions aimed at preventing the need for longterm care regarding oral function among the Japanese elderly.

Author Contributions: Conceptualization, K.M. and Y.W.; formal analysis, Y.M, M.I.; investigation, K.M., Y.W., M.S., A.E., Y.O ; data curation, Y.W., K.M., M.S., A.E., H.K ; writing-original draft preparation, K.M.; writing-review and editing, Y.W., K.M., M.S., H.K., A.E., Y.O., T.K., Y.F., K.I.; supervision, H.H., S.O.; project administration, Y.W., H.H, S.O.; funding acquisition, Y.W., H.H, S.O. All authors have read and agreed to the published version of the manuscript.

Funding: Please add: "This research received no external funding" or "This research was funded by Health Labour Sciences Research Grant（H23-Choju-Ippan-001、H23-Choju-Ippan-002、H25 - Choju-Ippan-005） .

Conflicts of Interest: The authors declare no conflict of interest.

\section{References}

1. Cabinet Office (2015) Annual report on the Aging Society Japanese 2015. Government of Japan. https://www8.cao.go.jp/kourei/whitepaper/w-2015/gaiyou/27pdf_indexg.html. Accessed 25 April 2019.

2. Ministry of Health, Labor and Welfare (2012) Health Japan 21 (the second term). Government of 
Japan. https://www.mhlw.go.jp/bunya/kenkou/dl/kenkounippon21_01.pdf. Accessed 25 April 2019.

3. Landi F, Zuccala G, Gambassi G, Incalzi RA, Manigrasso L, Pagano F, Carbonin P, Bernabei R (1999)

Body mass index and mortality among older people living in the community. J Am Geriatr Soc. doi:10.1111/j.1532-5415.1999.tb05229.x

4. Antonelli Incalzi R, Landi F, Cipriani L, Bruno E, Pagano F, Gemma A, Capparella O, Carbonin PU (1996) Nutritional assessment: A primary component of multidimensional geriatric assessment in the acute care setting. J Am Geriatr Soc. doi:10.1111/j.1532-5415.1996.tb02434.x

5. Incalzi RA, Gemma A, Capparella O, Cipriani L, Landi F, Carbonin P (1996) Energy intake and inhospital starvation: A clinically relevant relationship. Arch Intern Med. doi: 10.1001/archinte.1996.00440040101011

6. Tanaka T, Takahashi K, Hirano H, Kikutani T, Watanabe Y, Ohara Y, Furuya H, Tetsuo T, Akishita M, Iijima K (2018) Oral frailty as a risk factor for physical frailty and mortality in community-dwelling elderly. J Gerontol A Biol Sci Med Sci. doi: 10.1093/gerona/glx225

7. Tanimoto Y, Watanabe M, Sugiura Y, Hayashida I, Kusabiraki T, Kono K. Factors related to sarcopenia in community-dwelling elderly subjects in Japan. Nihon Koshu Eisei Zasshi 2013;60(11):683-690.

8. Iwasaki M, Yoshihara A, Ogawa H, Sato M, Muramatsu K, Watanabe R, Ansai T, Miyazaki H (2016) Longitudinal association of dentition status with dietary intake in Japanese adults aged 75 to 80 years. J Oral Rehabil. doi:10.1111/joor.12427

9. Zhu Y, Hollis JH (2015) Associations between the number of natural teeth and metabolic syndrome in adults. J Clin Periodontol. doi: 10.1111/jcpe.12361.

10. Koyano W, Hashimoto M, Fukawa T, Shibata H, Gunji A. Functional capacity of the elderly: measurement by the TMIG Index of Competence. Nihon Koshu Eisei Zasshi 1993;40(6):468-474.

11. Folstein MF, Folstein SE, McHugh PR (1975) “Mini-mental state". A practical method for grading the cognitive state of patients for the clinician. J Psychiatr Res. doi: 10.1016/0022-3956(75)90026-6

12. Kamiyama M, Kanazawa M, Fujinami Y, Minakuchi S (2010) Validity and reliability of a SelfImplementable method to evaluate masticatory performance: Use of color-changeable chewing gum and a color scale. J Prosthodont Res. doi: 10.1016/j.jpor.2009.08.001

13. Pifer TB, McCullough KP, Port FK, Goodkin DA, Maroni BJ, Held PJ, Young EW (2002) Mortality risk in hemodialysis patients and changes in nutritional indicators: DOPPS. Kidney Int. doi: 10.1046/j.15231755.2002.00658.x

14. Takahashi K, Yoshimura Y, Kaimoto T, Kunii D, Komatsu T, Yamamoto S (2001) Creation and validation of a food frequency questionnaire based on food groups for estimating individual nutrient intake. Jpn J Nutr Diet. doi: 10.5264/eiyogakuzashi.59.221

15. Willett W, Stampfer MJ (1986) Total energy intake: implications for epidemiologic analyses. Am J Epidemiol. doi: 10.1093/oxfordjournals.aje.a114366

16. Inomata C, Ikebe K, Kagawa R et al. (2014) Significance of occlusal force for dietary fibre and vitamin intakes in independently living 70-year-old Japanese: from SONIC Study. J Dent. doi:10.1016/j.jdent.2014.02.015

17. Ikuo Nasu, Saito Y (2012) A study of masticatory ability in the nationwide elderly population using a functional teeth triangle map. Jpn soc gerodontology. doi: 10.11259/jsg1987.16.204

18. Ministry of Health, Labour and Welfare (2016) Survey of dental diseases 2016. Government of Japan. https://www.mhlw.go.jp/toukei/list/62-28.html. Accessed 25 April 2019.

19. Usui Y, Miura H, Tamaki Y (2014) Survey on future needs and agendas related to promoting dental and 
oral health of super aging society among influential experts. Jpn J Gerodontology. doi: 10.11259/jsg.28.304

20. Haran PH, Rivas DA, Fielding RA (2002) Role and potential mechanisms of anabolic resistance in sarcopenia. J Cachexia Sarcopenia Muscle. doi: 10.1007/s13539-012-0068-4

21. Chevalier S, Gougeon R, Nayar K, Morais JA (2003) Frailty amplifies the effects of aging on protein metabolism: Role of protein intake. Am J Clin Nutr. doi:10.1093/ajcn/78.3.422.

22. Kumagai S, Watanabe S, Shibata H, Amano H, Fujiwara Y, Shinkai S, Yoshida H, Suzuki T, Yukawa H, Yasumura S, Haga H. Effects of dietary variety on declines in high-level functional capacity in elderly people living in a community. Nihon Koshu Eisei Zasshi 2003;50(12):1117-1124.

23. Motokawa K, Watanabe Y, Edahiro A et al. (2018) Frailty severity and dietary variety in Japanese older persons: A cross-sectional study. J Nutr Health Aging. doi:10.1007/s12603-018-1000-1.

24. Otsuka R, Nishita Y, Tange C, Tomida M, Kato Y, Nakamoto M, Imai T, Ando F, Shimokata H (2017) Dietary diversity decreases the risk of cognitive decline among Japanese older adults. Geriatr Gerontol Int. doi:10.1111/ggi.12817

25. Bradbury J, Thomason JM, Jepson NJA, Walls AWG, Allen PF, Moynihan PJ (2006) Nutrition counseling increases fruit and vegetable intake in the edentulous. J Dent Res. doi:10.1177/154405910608500513

26. Kikutani T, Yoneyama T, Teshima T, Horiuchi F, Miyatake M, Adachi M, Ishida M, Nishiwaki K, Tanaka K (2005) Effect of oral function training and dietary support on nutritional improvement in the elderly. Jpn J Gerodontology. doi:10.11259/jsg1987.20.208

27. Suzuki H, Kanazawa M, Komagamine Y, Iwaki M, Jo A, Amagai N, Minakuchi S (2018) The effect of new complete denture fabrication and simplified dietary advice on nutrient intake and masticatory function of edentulous elderly: A randomized-controlled trial. Clin Nutr. doi:10.1016/j.clnu.2017.07.022 